\title{
P38 MITOGEN-ACTIVATED PROTEIN KINASE AND PARKINSON'S DISEASE
}

Parkinson's disease, the second major neurodegenerative disease, has created a great impact on the elder people. Although the mechanisms underlying Parkinson's disease are not fully understood, considerable evidence suggests that neuro-inflammation, oxidative stress, mitochondrial dysfunction, cell proliferation, differentiation and apoptosis are involved in the disease. p38MAPK, an important member of the mitogen-activated protein family, controls several important functions in the cell, suggesting a potential pathogenic role in PD. This review provides a brief description of the role and mechanism of p38MAPK in Parkinson's disease.

Keywords Parkinson's disease $\cdot$ p38MAPK • neuro-inflammation • oxidative stress $•$ mitochondrial dysfunction

\section{Introduction}

Parkinson's disease (PD), the second most common neurodegenerative disease following Alzheimer's disease, has created a great impact on the elderly, the family and society. The average age of onset is about 60 years old, and the prevalence of Parkinson's disease in older people over 65 increases with age[1]. It is estimated that approximately 10 million individuals worldwide suffer from this disease, though many cases may go undiagnosed. With the growth of aging populations, the number will double over the next 25 years, which causes enormous social and economic problems.

The major pathological features of PD include progressive loss of dopaminergic (DA) neurons and formation of intracellular Lewy bodies (LBs) in the survival neurons of substantia nigra (SN). Its clinical manifestations include rest tremor, bradykinesia, muscle rigidity, posture gait abnormalities and other movement disorders, as well as cognitive disabilities, sleep disorders and other nonmotor barrier [2, 3], some of the symptoms of non-motor symptoms can occur prior to motor symptoms, and neurodegenerative neuropathies are not limited to SNc but have a broader impact[4, 5]. The etiology and pathogenesis of PD are complex and not yet fully understood. More studies suggest that genetic mutations in proteins play major role for the development and progression of $\mathrm{PD}[3,6]$. Neuro-inflammatory [7], oxidative stress[8], mitochondrial dysfunction[9] and cell proliferation, differentiation, apoptosis involve in the pathogenesis of both familial and idiopathic PD [10].

The MAPK cascade is a major intracellular signaling system that transmits extracellular information to the nucleus and mediates various cell responses and plays an significant role in cell proliferation, differentiation and apoptosis[11], it is one of the important signalregulated enzymes that connect the cell surface receptors with the decisive gene expression. p38 Mitogen-activated protein kinase (p38MAPK) is an important member of the mitogen-activated protein kinase family. The p38MAPK signaling cascade is a major signaling
Jianying $\mathrm{He}^{1,2}$,

Wenwen Zhong ${ }^{1,2}$,

Ming Zhang ${ }^{2}$,

Rongping Zhang ${ }^{1 *}$,

Weiyan $\mathrm{Hu}^{1,2,3^{*}}$

School of Pharmaceutical Science and Yunnan Key Laboratory of Pharmacology for Natural Products, Kunming Medical University, Kunming, China

${ }_{2}^{2}$ The Key Laboratory of Stem Cell and Regenerative Medicine, Institute of Molecular and Clinical Medicine, Kunming Medical University, Kunming, China

${ }^{3}$ Monash Immune Regeneration and Neuroscience Laboratories, Department of Anatomy and Developmental Biology, Monash University, Melbourne, Australia

Received 11 July 2018 accepted 08 August 2018 pathway for endogenous and endogenous stimulation (including growth factors, stress and cytokines) in respond to endothelial cell function and accordingly mediating a wide range of cellular effects, which provides cells with mechanisms to responding to external mitogenic signals[12-14]. p38MAPK play an important role in the pathogenesis of $\mathrm{PD}$.

\section{2. p38MAPK involves in neuro- inflammation in PD progression}

The PD patients showed accumulation of pro-inflammatory cytokines in the brain and cerebrospinal fluid, which demonstrates that neuro-inflammation is occurring in the affected brain area[15, 16]. In vivo evidence of neuropathic inflammation in PD patients includes cytokines and other molecular mediators expression disorders[17-19], microglia activation[20], peripheral immune cell invasion and changes around the composition and performance in Substantia nigra pars compacta (SNpc) [21]. Neuro-inflammation is thought to be an prominent pathological factor 
that contributes towards the development and progression of $\mathrm{PD}$ [22].

The development of neuro-inflammation plays an important role in the immune system of the central nervous system, which includes microglia and astrocytes[23]. The neuroinflammation process begins with the activation of glial cells, producing many neurotoxic components including reactive oxygen species (ROS), nitric oxide synthase (NOS), cytokines and other inflammatory mediators, all of which can lead to neurodegeneration[24, 25]. Inflammatory triggers such as $A \beta$, lipopolysaccharide (LPS) and MPTP can trigger inflammation and activate microglial cells. In addition to the generation of large amounts of free radicals after microglial activation, a large number of pro-inflammatory cytokines are released, such as IL-1 $\beta$, TNF- $\alpha$, TNF- $\gamma .[26,27]$. These inflammatory mediators can damage neurons and further activate microglial cells resulting in a vicious circle that aggravates neuro-inflammation and degeneration[28].

Activated microglia is observed in various degenerative neurological conditions such as PD and amyotrophic lateral sclerosis (ALS). Activated microglia can also increase ROS such as NO, superoxide Etc. As a result, these reactive substances can pass directly through the dopaminergic neurons against the endogenous antioxidant system and eventually cause oxidative stress and degeneration of dopaminergic neurons[29]. In addition, a series of enzymes, such as inducible nitric oxide synthase (iNOS) and cyclooxygenase (COX) 1 and 2 can be produced, which can cause some damage to dopaminergic neurons[30-32].

p38MAPK plays an important role in neuroinflammation and degeneration. Microglia reaction is the core of dopamine neuron degeneration, and recent studies have shown that p38MAPK signaling pathway plays a key role in microglial activation and response impact[33, 34]. Rotenone, dexmedetomidine and paraquat can all activate microglial cells by directly activating P38MAPK, which release large amounts of cytokines and thus damaging dopamine neurons[22, 35, 36]. These toxins can also induce NF- $\mathrm{KB}$ activation by directly activating P38MAPK, and iNOS expression is up-regulated. In glial cells, p38MAPK induces
iNOS to catalyze the production of nitric oxide (NO) in a large amount, excessive NO can cause lipid peroxidation and other nerve damage[37, 38], inhibiting the synthesis of DNA, leading to neuron death. It can also react with superoxide radicals to generate peroxynitrite and initiate a series of cytotoxicity, eventually leading to neuron loss [39].

In the 1-methyl-4-phenyl-1,2,3,6-tetrahydropyridine (MPTP) induced Parkinson's disease model mice, MPTP can induce the activation of p38 MAPK in the midbrain substantia nigra[40-42]. The activation of p38 leads to the phosphorylation of p38 and the increase of p-p38 leads to the up-regulation of cyclooxygenase-2 (COX-2), and the upregulation of COX-2 increases prostaglandin E2 (PGE2)[31, 40]. COX-2 overexpression, COX-2-mediated inflammatory response will further activate caspase-3, which results in dopaminergic neuron degeneration[43]. In addition, the high expression of COX-2 can induce inflammatory response, make reactive glial cell proliferation, increase the release of collagen damage $[43,44]$. COX-2 overexpression and its mediated inflammatory response involve in the oxidative stress response in the substantia nigra and cause damage to dopaminergic neurons $[45,46]$.

Lipopolysaccharide (LPS) is a major component of gram-negative bacterial cell walls and is now known to be an effective stimulator of macrophages in the brain. In vitro and in vivo studies have shown that LPS induced the activation of microglial cells leading to ROS, NOS and pro-inflammatory factors such

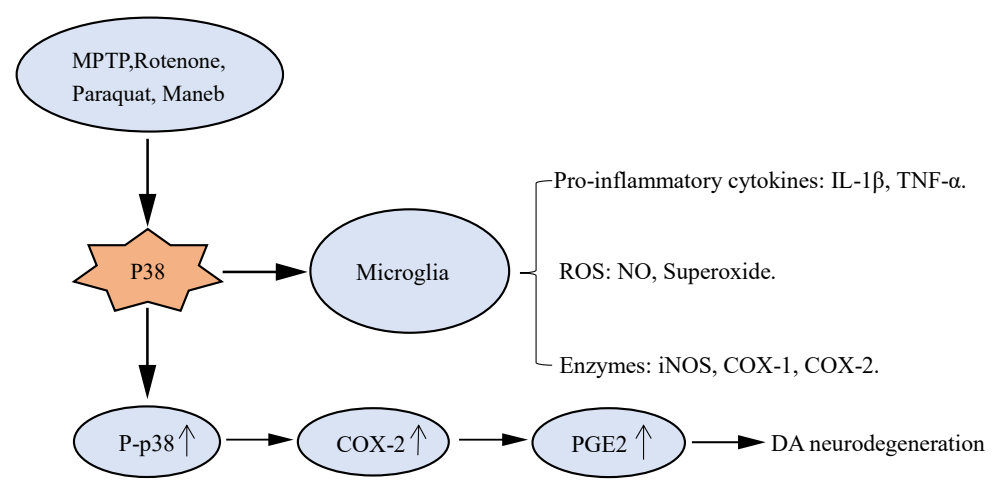

Fig 1. p38MAPK involves in neuro-inflammation in PD progression as IL-1 $\beta, I L-6$, TNF-a,IFNs production[47, 48]. p38 signaling cascade contributes to immunerelated cytotoxicity and neurodegenerative disease sequelae, in the LPS-induced PD model, LPS induces activation of the p38 and JNK pathways, which can increase IL-1 $\beta$, TNF- $a$ and the production of iNOS, which eventually leads to midbrain neuronal death. [24].

\section{3. p38MAPK acts in oxidative stress in PD development}

Oxygen is essential for all human life activities, and is crucial for all living cells. Oxidative stress exerts a causative role of in loss of dopamine neurons, which has been considered to be the pathological hallmark of PD. Genetic, environmental, drugs and other factors can induce oxidative stress response, triggering the body's redox reaction imbalance, resulting in dopamine neuron loss[49-51].

Oxidative stress triggers the p38 MAPK pathway, activating mitochondria and other mitochondrial apoptotic pathways in dopamine neurons. Paraquat, rotenone and MPTP all can directly or indirectly activate the p38MAPK pathway, resulting in increased accumulation of ROS $[35,52]$. On the other hand, activated p38MAPK can enhance the oxidative stress, making neurodegeneration[39].

Oxidative stress increases the steadystate levels of ROS and the ROS can regulate the activation of MAPKs in various stimulitriggered apoptosis[53,54], the production of ROS activates JNK and p38 MAPK[55], which can induce the production of ROS increased. 
Too much ROS can in turn affect the activation of p38MAPK, the formation of a feedback loop play an important role in the development of PD.

\section{4. p38MAPK makes an important role in mitochondrial dysfunction in PD occurrence}

Mitochondria play a vital role in energy metabolism. They provide a large amount of available energy in the form of mitochondrial ATP for intracellular metabolic pathways[56, 57]. Mitochondria are highly dynamic, multifunctional organelles, in addition to their primary role in energy metabolism, they are also essential for many cellular processes including neurotransmission, synaptic maintenance, calcium homeostasis, cell death and neuronal survival[58, 59].

Mitochondrial dysfunction is a common feature of sporadic and familial PD. The main manifestations of mitochondrial dysfunction include ROS production, mitochondrial electron transport complex enzymatic activity defection, ATP depletion, caspase-3 release and mitochondrial DNA consumption[60, 61]. Inhibition of mitochondrial complex I or blockade of normal electron transfer may lead to ROS increase and ATP decrease[58], which may damage mitochondrial DNA, destroy respiratory chain and triggering a vicious cycle between mitochondrial damage and oxidation[4].

Energy failure, oxidative stress, genetic mutations and environmental toxicants in PD are closely linked to mitochondrial dysfunction[61]. Neurotoxins such as MPTP, rotenone and paraquat induce the death of dopamine neurons directly related to the mitochondrial complex I activity inhibition, which in turn may cause different mitochondrial disorders and subsequently neuronal degeneration[60, 62].

Mitochondria metabolism is the major sources for ROS that may contribute to intracellular oxidative stress, mitochondrial respiratory chain disorder, particularly complex I deficiency, and the increase of ROS may directly or indirectly lead to the production of sporadic PD[63-65]. Existing research shows that ROS can regulate intracellular signal cascades. Excessive ROS production can lead to intracellular stimulation and mitochondria damage, eventually leading to apoptosis and necrosis.

MPTP, rotenone and paraquat can cause mitochondrial dysfunction, triggering other stimuli in neurons[42, 66]. MPTP is selectively

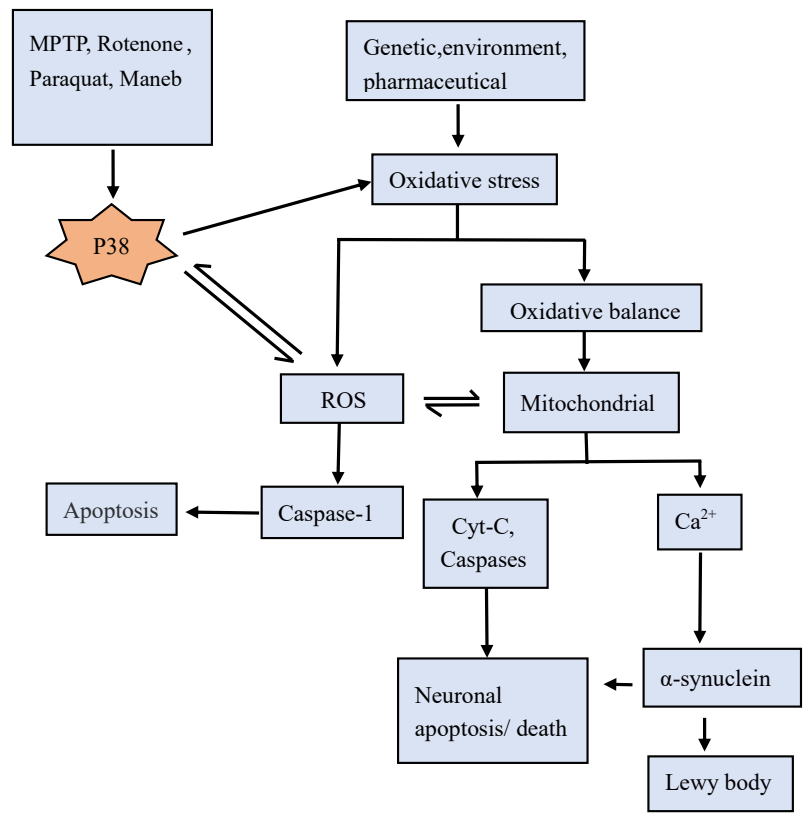

Fig 2. p38MAPK acts in oxidative stress in PD development

toxic to dopaminergic neurons, it can cross the blood-brain barrier in minutes and is rapidly metabolized by monoamine oxidase $B$ (MAOB) to the active metabolite MPP+ in the brain, which is selectively transported to dopaminergic neurons[67], then accumulates in the mitochondria[68,69]. MPP+, an active metabolite in mitochondria, suppresses mitochondrial complex $\mathbf{I}$ in the electron transport chain, thereby disrupting the flow of electrons, leading to a decrease in ATP production and an increase in ROS production[68-70]. The expression of MAOB is regulated by the activation of $\mathrm{p} 38$ MAPK, and the activation of p38 MAPK is accompanied by astrocyte proliferation, and then causes astrocytes and neuron loss. The activation of MAOB can be prevented by inhibiting the p38 MAPK pathway[67, 71, 72].

One possibility that cytoplasmic p38 affects mitochondria is that p38 activation induces the translocation of its substrate ( $p 53)$ into mitochondria, which in turn eliminates unhealthy mitochondrial proteins and thereby protect mitochondrial dysfunction[73, 74].

On the other hand, activating the p38MAPK pathway may indirectly induce the mitochondrial pro-apoptotic protein Bax to produce CytC by activating p53, and CytC can activate caspase- 3 and cause apoptosis of dopamine neurons [49, 58, 75]. In the study done by Fengsen Duan, ROS was found to regulate the expression of $\mathrm{p} 38 \mathrm{MAPK}$, eventually resulting in mitochondrial damage, which fed back each other and formed a vicious circle[55].

Mitochondrial dysfunction splays an important role in PD occurrence, progression and development. Currently there are many substances against mitochondrial damage used in PD treatment, such as antioxidant enzymes (SOD, CAT), a-lipoic acid, green tea polyphenols, melatonin, ginseng water extract, all showed an improved effect against PD[76, 77].

\section{Conclusion}

Parkinson's disease affects approximately $1-2 \%$ of the population over 65 years of age, and up to $5 \%$ of the population by age 85 . Though efforts have been made to elucidate 
the PD pathogenesis, the mechanisms are still not understood clearly. Neuro-inflammation, oxidative stress can accelerate the progress and development of Parkinson's disease. Mitochondrial dysfunction plays an important role in the occurrence of $\mathrm{PD}$ [39].

p38, as a key member of the signal transduction pathways, plays a crucial role during the process of apoptosis. More and more evidence has shown that the activation of p38 MAPK signal pathway has a vital role in promoting the development of PD and the inhibitory effect of p38 can appropriately improve the therapeutic effect of PD, which may provide a new medicinal strategy for the treatment of PD, this pathway can be used as a breakthrough in the study of Parkinson's disease, and then find effective control disease treatment. In vitro experimental studies showed that minocycline could prevent NOinduced phosphorylation of $\mathrm{p} 38$ and cell death associated with NO-induced toxicity, which was neuroprotective in many neurodegenerative models, such as the 1-methyl-4-phenyl-1, 2,3,6-hydrogen pyridine (MPTP) model of $\mathrm{PD}[78,79]$

Although there are many researches and some medicines have a therapeutic effect on Parkinson's disease, none of them cure the disease fundamentally. Neuro-inflammation, oxidative stress and mitochondrial dysfunction in PD are closely liked to P38MAPK, it may be

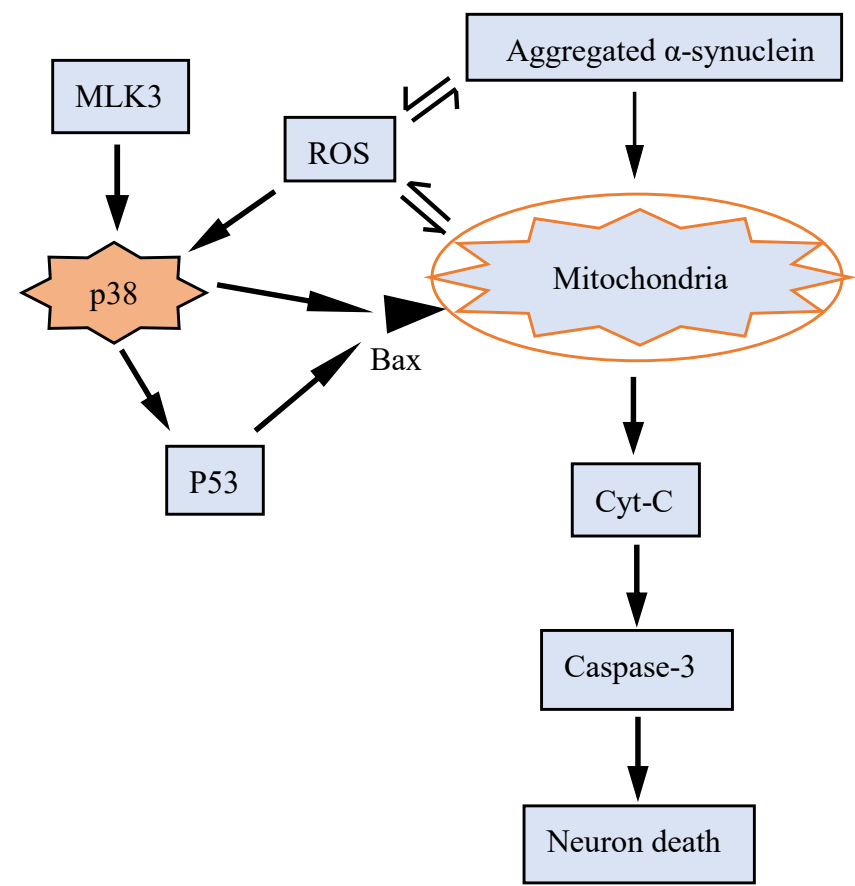

Fig 3. p38MAPK makes an important role in mitochondrial in PD occurrence

a target to PD. So for best understanding this signal pathway in PD occurrence progress and development is essential[80].

\section{Acknowledgements}

This work was supported by the fund of Yunnan key program of Science and
Technology (2014FA031), National Natural Science Foundation of China (81460593) and Yunnan program of Science and Technology (2015FB006).

\section{References}

[1] Rijk M C De, C. Tzourio, M M Breteler, J F Dartigues, L. Amaducci, S. Lopezpousa, J M Manubensbertran, A. Alpérovitch, W A Rocca. Prevalence of parkinsonism and Parkinson's disease in Europe: the EUROPARKINSON Collaborative Study. European Community Concerted Action on the Epidemiology of Parkinson's disease[J], Journal of Neurology Neurosurgery \& Psychiatry, 1997, 62, 10-5

[2] Zhangna, Ke Kai Fu, Qiu Yihua, Peng Yu-Ping. Role of neuroinflammation in the pathogenesis of Parkinson's disease[J], Transportation Medicine, 201256-58+62

[3] Xu Y., Y. Deng, H Qing. The phosphorylation of a-synuclein: development and implication for the mechanism and therapy of the Parkinson's disease[J], J. Neurochem., 2015, 135, 4-18

[4] Exner Nicole, Anne Kathrin Lutz, Christian Haass, Konstanze F Winklhofer. Mitochondrial dysfunction in Parkinson's disease: molecular mechanisms and pathophysiological consequences[J], EMBO J., 2012, 31, 3038
[5] Solla P., A. Cannas, G. L. Floris, G. Orofino, E. Costantino, A. Boi, C. Serra, M. G. Marrosu, F. Marrosu. Behavioral, neuropsychiatric and cognitive disorders in Parkinson's disease patients with and without motor complications[J], Prog. Neuro-Psychopharmacol. Biol. Psychiatry, 2011, 35, 1009-1013

[6] Zuo L., M. S. Motherwell. The impact of reactive oxygen species and genetic mitochondrial mutations in Parkinson's disease[J], Gene, 2013, 532, 18-23

[7] Whitton P. S., Inflammation as a causative factor in the aetiology of Parkinson's disease[J], Br. J. Pharmacol., 2007, 150, 963-976

[8] Dias Vera, Eunsung Junn, M. Maral Mouradian. The Role of Oxidative Stress in Parkinson's Disease[J], Journal of Parkinsons Disease, 2013, 3, 461-491

[9] Wanglund C., D. Lynch, R. Spathis, J. K. Lum, R. M. Garruto. Evidence for mitochondrial dysfunction and its role in neurodegeneration in Guam ALS and PD[J], Amer. J. Hum. Biol., 2006, 18, 280-280 
[10] Sheng-Gang Sun, Li Gang, Cao Xuebing, Tong Calyx Tong. Research Progress on the Relationship between Parkinson 's Disease and Inflammatory Reaction[J], Chinese Journal of Neurology, 20034-6

[11] Junli Zhen, Wang Weiping, An Liwei, Li Zhou Ping, Jia Li King. Progress in research of p38 mitogen - activated protein kinase pathway and its related nervous system[J], Journal of Brain and Nervous Diseases, 2010318-321

[12] Corre I, F Paris, J Huot. The p38 pathway, a major pleiotropic cascade that transduces stress and metastatic signals in endothelial cells[J], Oncotarget, 2017, 8, 55684-55714

[13] Jin-Tao L. I., Tin Hua Wang, L. I. Li-Yan. Role of P38, a Kind of Signaling Molecules in CNS Diseases[J], Journal of Kunming Medical University, 2012

[14] Coulthard Lydia R., Danielle E. White, Dominic L. Jones, Michael F. Mcdermott, Susan A. Burchill. p38MAPK: stress responses from molecular mechanisms to therapeutics[J], Trends Mol. Med., 2009, 15,369

[15] Tiwari P. C., R. Pal. The potential role of neuroinflammation and transcription factors in Parkinson disease[J], Dialogues Clin. Neurosci., 2017, 19, 71-80

[16] Przedborski Serge. Neuroinflammation and Parkinson's disease[J], Handb. Clin. Neurol., 2007, 83, 535

[17] Mogi Makio, Minoru Harada, Tomoyshi Kondo, Peter Riederer, Hirofumi Inagaki, Masayasu Minami, Toshiharu Nagatsu. Interleukin$1 \beta$, interleukin-6, epidermal growth factor and transforming growth factor- $a$ are elevated in the brain from parkinsonian patients[J], Neurosci. Lett., 1994, 180, 147-150

[18] Nagatsu T, M Mogi, H Ichinose, A Togari. Changes in cytokines and neurotrophins in Parkinson's disease[J], J. Neural Transm. Suppl., 2000, 80, 277-90

[19] Depino A. M., C. Earl, E. Kaczmarczyk, C. Ferrari, H. Besedovsky, A Rey Del, F. J. Pitossi, W. H. Oertel. Microglial activation with atypical proinflammatory cytokine expression in a rat model of Parkinson's disease[J], Eur. J. Neurosci., 2003, 18, 2731-42

[20] Mcgeer P. L., S. Itagaki, B. E. Boyes, E. G. Mcgeer. Reactive microglia are positive for HLA-DR in the substantia nigra of Parkinson's and Alzheimer's disease brains[J], Neurology, 1988, 38, 1285

[21] Mcgeer P. L., S Itagaki, H Akiyama, E. G. Mcgeer. Rate of cell death in parkinsonism indicates active neuropathological process[J], Ann. Neurol., 1988, 24, 574-576

[22] Chao Y., S. C. Wong, E. K. Tan. Evidence of inflammatory system involvement in Parkinson's disease[J], Biomed Research International, 2014, 2014, 308654

[23] Skaper S. D., The brain as a target for inflammatory processes and neuroprotective strategies[J], Ann. N. Y. Acad. Sci., 2007, 1122, 23-34

[24] Badshah H, T Ali, Shafiq Ur Rehman, Faiz UI Amin, F Ullah, T. H. Kim, M. O. Kim. Protective Effect of Lupeol Against LipopolysaccharideInduced Neuroinflammation via the p38/c-Jun N-Terminal Kinase Pathway in the Adult Mouse Brain[J], J. Neuroimmune Pharmacol., 2016, 11, 48

[25] Hirsch E. C., S Hunot, P Damier, B Faucheux. Glial cells and inflammation in Parkinson's disease: a role in neurodegeneration?[J], Ann. Neurol., 1998, 44, 115-20

[26] Duty Susan, Peter Jenner. Animal models of Parkinson's disease: a source of novel treatments and clues to the cause of the disease[J], Br. J. Pharmacol., 2011, 164, 1357-1391

[27] Mosley R. Lee, Eric J. Benner, Irena Kadiu, Mark Thomas, Michael D. Boska, Khader Hasan, Chad Laurie, Howard E. Gendelman. Neuroinflammation, Oxidative Stress and the Pathogenesis of Parkinson's Disease[J], Clin. Neurosci. Res., 2006, 6, 261-281

[28] Wang X. H., X. Xie, X. G. Luo, H. Shang, Z. Y. He. Inhibiting purinergic $P 2 X 7$ receptors with the antagonist brilliant blue $G$ is neuroprotective in an intranigral lipopolysaccharide animal model of Parkinson's disease[J], Mol. Med. Report., 2017, 15, 768-776

[29] Appel Stanley H. Beers David R. , Henkel Jenny S. T cell-microglial dialogue in Parkinson's disease and amyotrophic lateral sclerosis: are we listening?[J], Trends Immunol., 2010, 31, 7-17

[30] Knott C, G Stern, G. P. Wilkin. Inflammatory regulators in Parkinson's disease: iNOS, lipocortin-1, and cyclooxygenases-1 and -2[J], Molecular \& Cellular Neuroscience, 2000, 16, 724-739

[31] Teismann Peter, Boris Ferger. Inhibition of the cyclooxygenase isoenzymes COX- 1 and COX-2 provide neuroprotection in the MPTPmouse model of Parkinson's disease[J], Synapse, 2001, 39, 167

[32] Li M., F. R. Dai, X. P. Du, Q. D. Yang, Y. Chen. Neuroprotection by silencing iNOS expression in a 6-OHDA model of Parkinson's disease[J], Journal of Molecular Neuroscience Mn, 2012, 48, 225-33

[33] Gang Li, Ma Rong, Sun Sheng-Gang, Tong Calyx Tong. Role of p38 MAPK pathway in the degeneration of dopaminergic neurons mediated by microglial activation[J], Journal of Brain and Nervous Diseases, 2006, 14, 105-107

[34] Wilms H. Rosenstiel P, J Sievers, G Deuschl, L Zecca, R Lucius. Activation of microglia by human neuromelanin is NF-kappa B-dependent and involves p38 mitogen-activated protein kinase: implications for Parkinson's disease[J], FASEB J., 2003, 17, 500

[35] Gao F., D. Chen, Q. Hu, G. Wang. Rotenone directly induces BV2 cell activation via the p38 MAPK pathway[J], PLoS One, 2013, 8, e72046

[36] Pei Yinghao, Xiaomin Cai, Jiao Chen, Baodi Sun, Zhaorui Sun, Xing Wang, Xiaomin Qian. The role of p38 MAPK in acute paraquatinduced lung injury in rats[J], Inhalation Toxicol., 2014, 26, 880-4

[37] Qian Wang, Zhang Hui, Liu Name, Zhang Zuofeng, Wei Zifeng, Sunna, Mao Tong Yao, Zhang Yu New. P38 Signal Pathway Regulates NFKB and iNOS Expression in Parkinson's Disease Substantia Nigra[J], Journal of Southern Medical University, 2014, 34, 1176-1180

[38] Qian Wang, Zhang Hui, Zhang Zuofeng, Wei Zifeng, Wang Yongsheng, Zhou Hongxia, Zhang Yu New. Regulation of P38MAPK on NF-KB and COX-2 in Parkinson's Disease MPTP Model Mice[J], China Journal of Modern Medicine, 2012, 22, 15-20

[39] Jha S. K., N. K. Jha, R. Kar, R. K. Ambasta, P. Kumar. p38 MAPK and PI3K/ AKT Signalling Cascades inParkinson's Disease[J], Int J Mol Cell Med, 2015, 4, 67-86

[40] Qian Wang, Zheng Huan, Zhang Zuofeng, Zhang Yu New. Ginsenoside Rg1 Affects the Expression of COX-2 in the substantia nigra of MPTP 
Model of Parkinson's Disease through P38 Signal Pathway[J], Journal of Southern Medical University, 2008, 28, 1594-1598

[41] Hwang Chul Ju, Hee Pom Lee, Dong Young Choi, Heon Sang Jeong, Tae Hoon Kim, Tae Hyung Lee, Young Min Kim, Dae Bong Moon, Sung Sik Park, Young Kim Sun. Inhibitory effect of thiacremonone on MPTP-induced dopaminergic neurodegeneration through inhibition of p38 activation[J], Oncotarget, 2016, 7, 46943-46958

[42] Ray A, N Sehgal, S Karunakaran, G Rangarajan, V Ravindranath. MPTP activates ASK1-p38 MAPK signaling pathway through TNFdependent Trx1 oxidation in parkinsonism mouse model[J], Free Radic. Biol. Med., 2015, 87, 312-325

[43] Fahn Stanley, David Sulzer. Neurodegeneration and Neuroprotection in Parkinson Disease[J], NeuroRx, 2004, 1, 139-154

[44] Bright Teacher, Zhang Yu New, Zhang Zuofeng, Chen Hao. Toxicity of COX-2 to dopaminergic neurons in the substantia nigra of Parkinson's disease mice[J], Medical debate, 2006, 27, 1661-1664

[45] Teismann Peter, Kim Tieu, Dongkug Choi, Duchu Wu, Ali Naini, Stéphane Hunot, Miquel Vila, Vernice Jacksonlewis, Serge Przedborski. Cyclooxygenase- 2 is instrumental in Parkinson's disease neurodegeneration[J], Proc Natl Acad Sci U S A., 2003, 100, 54735478

[46] Hald A, J Lotharius. Oxidative stress and inflammation in Parkinson's disease: is there a causal link?[J], Exp. Neurol., 2005, 193, 279-290

[47] Pålsson-Mcdermott E. M., L. A. O'neill. Signal transduction by the lipopolysaccharide receptor, Toll-like receptor-4[J], Immunology, 2004, 113, 153-162

[48] Xaio Haipeng, W. A Banks, Michael L Niehoff, J. E Morley. Effect of LPS on the permeability of the blood-brain barrier to insulin[J], Brain Res., 2001, 896, 36

[49] Kim E. K., E. J. Choi. Pathological roles of MAPK signaling pathways in human diseases[J], Biochim. Biophys. Acta, 2010, 1802, 396-405

[50] Jenner Peter, C. Warren Olanow. Oxidative stress and the pathogenesis of Parkinson's disease[J], Neurology, 1996, 47, 161-70

[51] Varçin M, E Bentea, Y Michotte, S Sarre. Oxidative Stress in Genetic Mouse Models of Parkinson's Disease[J], Oxid. Med. Cell. Longev., 2012, 2012, 624925

[52] Karunakaran S, V Ravindranath. Activation of p38 MAPK in the substantia nigra leads to nuclear translocation of NF-kappaB in MPTPtreated mice: implication in Parkinson's disease[J], J. Neurochem., 2009, 109, 1791-1799

[53] Santabárbararuiz P, M Lópezsantillán, I Martínezrodríguez, A Binaguicasas, L Pérez, M Milán, M Corominas, F Serras. ROS-Induced JNK and p38 Signaling Is Required for Unpaired Cytokine Activation during Drosophila Regeneration[J], PLoS Genet., 2015, 11, e1005595

[54] Dunn Laura, Vanessa Fairfield, Shanay Daham, Juan P. Bolaños, Simon J. Heales. Pentose-phosphate pathway disruption in the pathogenesis of Parkinson's disease[J], Transl. Neurosci., 2014, 5, 179184

[55] Duan F., Y. Yu, R. Guan, Z. Xu, H. Liang, L. Hong. Vitamin K2 Induces Mitochondria-Related Apoptosis in Human Bladder Cancer Cells via ROS and JNK/p38 MAPK Signal Pathways[J], PLoS One, 2016, 11, e0161886

[56] Ju X., Y. Wen, D Metzger, M Jung. The role of p38 in mitochondrial respiration in male and female mice[J], Neurosci. Lett., 2013, 544, 152-156

[57] Yao J., R. W. Irwin, L. Zhao, J Nilsen, R. T. Hamilton, R. D. Brinton. Mitochondrial bioenergetic deficit precedes Alzheimer's pathology in female mouse model of Alzheimer's disease[J], Proc. Natl. Acad. Sci. U. S. A., 2009, 106, 14670

[58] Trancikova Alzbeta, Elpida Tsika, Darren J. Moore. Mitochondrial Dysfunction in Genetic Animal Models of Parkinson's Disease[J], Antioxidants \& Redox Signaling, 2012, 16, 896

[59] Suzuki M, N Motizuki, T Koya, A Kitabatake. [Mitochondrial disorders] [J], J. Pediatr. Neurol., 2009, 49, 27

[60] Luo Yu, Alan Hoffer, Barry Hoffer, Xin Qi. Mitochondria: A Therapeutic Target for Parkinson's Disease?[J], Int. J. Mol. Sci., 2015, 16, 2070420730

[61] Helley Martin P., Jennifer Pinnell, Carolina Sportelli, Kim Tieu. Mitochondria: A Common Target for Genetic Mutations and Environmental Toxicants in Parkinson's Disease[J], Frontiers in Genetics, 2017, 8, 177

[62] Votyakova Tatyana V., Ian J. Reynolds, Mitochondrial Complex I Deficiency in Parkinson's Disease: A Mechanism for Oxidant-Based Pathogenesis[M], 2007.

[63] Onyango Isaac G., Mitochondrial Dysfunction and Oxidative Stress in Parkinson's Disease[J], Prog. Neurobiol., 2013, 106-107, 17

[64] Zorov D. B., M Juhaszova, S. J. Sollott. Mitochondrial ROS-induced ROS release: an update and review[J], Biochim. Biophys. Acta, 2006, 1757, 509-517

[65] Jellinger Kurt A., The role of a-synuclein in neurodegeneration - An update[J], Transl. Neurosci., 2012, 3, 75-122

[66] Langston J. William, lan Irwin, Elizabeth B. Langston, Lysia S. Forno. 1-Methyl-4-phenylpyridinium ion (MPP + ): Identification of a metabolite of MPTP, a toxin selective to the substantia nigra[J], Neurosci. Lett., 1984, 48, 87-92

[67] Hwang C. J., Choi D Young, Y. Y. Jung, Y. J. Lee, J. S. Yun, K. W. Oh, S. B. Han, S Oh, M. H. Park, J. T. Hong. Inhibition of p38 pathwaydependent MPTP-induced dopaminergic neurodegeneration in estrogen receptor alpha knockout mice[J], Horm. Behav., 2016, 80, 19-29

[68] Nicklas W. J., I Vyas, R. E. Heikkila. Inhibition of NADH-linked oxidation in brain mitochondria by 1-methyl-4-phenyl-pyridine, a metabolite of the neurotoxin, 1-methyl-4-phenyl-1,2,5,6-tetrahydropyridine[J], Life Sci., 1985, 36, 2503

[69] Hasegawa E, K Takeshige, T Oishi, Y Murai, S Minakami. 1-Methyl-4phenylpyridinium (MPP+) induces $\mathrm{NADH}$-dependent superoxide formation and enhances $\mathrm{NADH}$-dependent lipid peroxidation in bovine heart submitochondrial particles[J], Biochem. Biophys. Res. Commun., 1990, 170, 1049-55

[70] Chan P., L. E. Delanney, I Irwin, J. W. Langston, Monte D Di. Rapid ATP loss caused by 1-methyl-4-phenyl-1,2,3,6-tetrahydropyridine in mouse brain[J], J. Neurochem., 2010, 57, 348-351 
[71] Wong Wai K., Xiao Ming Out, Kevin Chen, Jean C. Shih. Activation of Human Monoamine Oxidase B Gene Expression by a Protein Kinase C MAPK Signal Transduction Pathway Involves c-Jun and Egr-1[J], J. Biol. Chem., 2002, 277, 22222-22230

[72] Roy Choudhury G, M. G. Ryou, E Poteet, Y. Wen, R. He, F. Sun, F. Yuan, K. Jin, S. H. Yang. Involvement of p38 MAPK in reactive astrogliosis induced by ischemic stroke[J], Brain Res., 2014, 1551, 45-58

[73] Kitamura N, Y Nakamura, Y Miyamoto, T Miyamoto, K Kabu, M Yoshida, M Futamura, S Ichinose, H Arakawa. Mieap, a p53-Inducible Protein, Controls Mitochondrial Quality by Repairing or Eliminating Unhealthy Mitochondria[J], PLoS One, 2011, 6, e16060

[74] M Gomez-Lazaro, Galindo Mf, Concannon Cg, Segura Mf, Fernandez-Gomez Fj, Llecha N, Comella Jx, Prehn Jh, Jordan J. 6-Hydroxydopamine activates the mitochondrial apoptosis pathway through p38 MAPK-mediated, p53-independent activation of Bax and PUMA[J], J. Neurochem., 2008, 104, 1599-1612

[75] Das N. R., S. S. Sharma. Cognitive Impairment Associated with
Parkinson's Disease: Role of Mitochondria[J], Curr. Neuropharmacol., 2016, 14, -

[76] FernÃ $£$ Ndez-Moriano C, Lez-Burgos E GonzÃ£, M. P. GÃ£ẨmezSerranillos. Mitochondria-Targeted Protective Compounds in Parkinson's and Alzheimer's Diseases[J], Oxid. Med. Cell. Longev., 2015, 2015, 408927

[77] Absi E, A Ayala, A Machado, J Parrado. Protective effect of melatonin against the 1-methyl-4-phenylpyridinium-induced inhibition of complex I of the mitochondrial respiratory chain[J], J. Pineal Res., 2010, 29, 40-47

[78] Harper Sarah J, Neil Wilkie. MAPKs: new targets for neurodegeneration[J], Expert Opin. Ther. Targets, 2003, 7, 187

[79] Thomas M, W. D. Le. Minocycline: neuroprotective mechanisms in Parkinson's disease[J], Curr. Pharm. Des., 2004, 10, 679-686

[80] Koul Hari K., Mintu Pal, Sweaty Koul. Role of p38 MAP Kinase Signal Transduction in Solid Tumors[J], Genes Cancer, 2013, 4, 342 